\title{
Sleep-related breathing disorder is associated with hyperactivity in preschoolers
}

Zhimin $\underline{\operatorname{Ren}}^{1}, \mathrm{MD}$, Anli $\underline{\mathrm{Qiu}}^{2}, \mathrm{MD}$

INTRODUCTION Sleep problems are a prominent feature in children with attention deficit hyperactivity disorder (ADHD). Unlike existing studies that focused on extreme samples (i.e. normal vs. ADHD), our study investigated the associations of sleep-related behaviours and ADHD features in nonclinical Chinese preschoolers.

METHODS All participants were recruited via advertisements and screened for eligibility through a telephone interview prior to an onsite visit. The maternal reports of the Conners' Parent Rating Scale (CPRS) and Pediatric Sleep Questionnaire (PSQ) were acquired from 110 Chinese preschoolers aged six years. Regression models were used to examine the association between CPRS and PSQ scores.

RESULTS The results obtained from regression models on the CPRS and PSQ scores of the 110 participants showed that none of the sleep-related behavioural measures (i.e. sleep-related breathing disorder [SRBD], snoring, daytime sleepiness, restless legs syndrome) was associated with inattention in our sample. However, worse SRBD was associated with higher hyperactivity.

CONCLUSION Our study underpins the importance of understanding the relationship between sleep-related behaviours and ADHD characteristics before the usual age of clinical diagnosis in children with ADHD.

Keywords: ADHD, Conners' Parent Rating Scale, hyperactivity, inattention, sleep-related breathing disorder

\section{INTRODUCTION}

Attention deficit hyperactivity disorder (ADHD) is one of the most common neurodevelopmental disorders, with an estimated worldwide prevalence of approximately $5 \%$ in school-age children. ${ }^{(1)}$ It is characterised by developmentally inappropriate symptoms of inattention, hyperactivity and/or impulsivity, with the onset before the age of seven years. ${ }^{(2)}$

Sleep problems are a prominent feature in children with ADHD. ${ }^{(3-7)}$ Based on parental reports, sleep-related problems such as sleep-related breathing disorder (SRBD) and restless legs syndrome were more often reported in children with ADHD than in control children. ${ }^{(3-7)}$ Using polysomnography and actigraphy, disturbances in sleep architecture can be found in ADHD children. However, there is no specific pattern of disturbance related to ADHD. ${ }^{(8)}$ Recently, Scott et al showed that shorter sleep duration and sleep disturbances appear early and predate the usual age of clinical diagnosis in children with $\mathrm{ADHD}^{\left({ }^{(9)}\right.}$ suggesting the importance of understanding the role of sleeprelated behaviours in the early prediction of ADHD. Sadeh et al suggested that possible underlying involvement of sleep disorders or insufficient sleep should be considered when performing diagnostic procedures in children suspected to have ADHD. ${ }^{(5)}$

While there is a wealth of studies on sleep patterns in children with ADHD, few studies focused on nonclinical samples of preschoolers or early school-age children, or investigated the direct link between sleep-related phenomena and the cardinal features of ADHD (e.g. inattention and hyperactivity/ impulsivity). In addition, most of the existing studies on this topic have been limited to Western populations, whereas only a few studies on Chinese children have been reported. In school-age Chinese children, sleep-disordered breathing problems, daytime inadvertent napping and sleep schedules were found to be linked to ADHD-related symptoms. ${ }^{(10)}$

The aim of this study was to examine the association between sleep-related behaviours and the cardinal features of ADHD in a nonclinical sample of six-year-old Chinese boys. This sample was selected based on emerging evidence supporting a dimensional view of ADHD, suggesting that ADHD symptoms lie on a continuum. ${ }^{(11)}$ If indeed sleep problems identified in studies with extreme samples (i.e. normal vs. ADHD subjects) are directly associated with these cardinal features of ADHD, then we could expect this relation to hold across a continuum defined by inattention and/or hyperactivity/impulsivity. As the study of ADHD in young children is in part constrained by the relative lack of opportunity for the observation of such behaviours in a controlled environment such as a school setting, clinical assessment of ADHD features in young children is therefore highly dependent upon parental reports. Such reports are compromised by parental attitudes and mental health. Hence, we investigated the relationship between maternal reports on the Conners' Parent Rating Scale (CPRS) and the Pediatric Sleep Questionnaire (PSQ). Our study recruited boys, as previous studies demonstrated a higher prevalence of ADHD in boys than in girls. ${ }^{(12,13)}$ Moreover, our study only incorporated CPRS and PSQ ratings obtained from mothers, who are generally the primary custodians of children within the family. ADHD children

${ }^{1}$ Department of Pediatrics, The Second Affiliated Hospital of Harbin Medical University, ${ }^{2}$ Department of Respiratory Medicine, Harbin Children's Hospital, Harbin, China Correspondence: Dr Zhimin Ren, Consultant, The Second Affiliated Hospital of Harbin Medical University, 148 Baojianlu, Nangang District, Harbin 150086, China. rrzzmm1966@sina.com 
appear to be more compliant and less disruptive when they are with their fathers than with their mothers. ${ }^{(14)}$

\section{METHODS}

A total of 110 Chinese boys aged $72-79$ (mean \pm standard deviation $[\mathrm{SD}] 75.6 \pm 2.3$ ) months and their mothers participated in this study. The participants were recruited via advertisements and screened for eligibility through a telephone interview prior to an onsite visit. We excluded boys with an existing diagnosis of chronic medical conditions (e.g. cancer, respiratory problems, congenital abnormalities) and/or mental illnesses (e.g. ADHD, autism). Boys whose mothers reported adverse pregnancy indicators (e.g. smoking, alcohol consumption, gestational complications) were also excluded from the study. Written consent was obtained from the participants' parents.

The short form of the CPRS, which consists of 27 items concerning a variety of childhood behavioural problems, ${ }^{(15)}$ was used in this study. Mothers were asked to rate each item according to their child's behaviour over the last month on a four-point scale ( 1 = never, seldom; 2 = occasionally; 3 = often, quite a bit; and 4 = very often, very frequent). Ratings were summed up according to the appropriate dimension, resulting in four subscale scores - opposition, inattention, hyperactivity/ impulsivity and ADHD Index. The CPRS subscales of inattention, hyperactivity/impulsivity and opposition were then standardised into T-scores to reflect the levels of inattention, hyperactivity/ impulsivity and opposition.

The modified version of the PSQ consists of 40 items and provides four sleep scales: (a) SRBD; (b) snoring; (c) sleepiness; and (d) restless legs syndrome (RLS). This version has been well validated for the Chinese population. ${ }^{(16)}$ Mothers were asked to rate each item according to their child's usual sleep habits currently or in the last two years based on responses to yes/no questionnaire format; the responses 'does not apply' and 'applies just a little' were scored as 'no' (i.e. score 0), and the responses 'applies quite a bit' and 'definitely applies most of the time' were scored as 'yes' (i.e. score 1). Ratings were summed up according to the appropriate dimension and then normalised by the total score of this dimension, resulting in the aforementioned four sleep scale scores. Both questionnaires were completed in the predominant language used by the population, which was Chinese.

Statistical analysis was performed using the Statistical Package for the Social Sciences version 19 (SPSS Inc, Chicago, IL, USA). A series of multiple regression analyses was used to examine which sleep scale associates with the CPRS subscales of inattention, hyperactivity/impulsivity and opposition. For this, the four sleep scales were simultaneously entered as the primary independent variables. Separate models evaluated each CPRS scale as a dependent variable.

\section{RESULTS}

In our sample, the range of the CPRS inattention score was 41.581.6 and the range of the CPRS hyperactivity/impulsivity score
Table I. Associations between sleep-related behaviours and ADHD features.

\begin{tabular}{lcccc}
\hline ADHD feature & SRBD & Snoring & Sleepiness & RLS \\
\hline Inattention & 0.273 & 0.161 & -0.026 & -0.046 \\
Hyperactivity/impulsivity & $0.687^{*}$ & -0.224 & $-0.253^{+}$ & 0.119 \\
Opposition & 0.179 & 0.134 & 0.034 & 0.088 \\
\hline
\end{tabular}

Note: Standardised coefficient values indicate which sleep measures are associated with the inattention, hyperactivity/impulsivity and opposition scores of the Conners' Parent Rating Scale.

${ }^{*} p<0.005 ;{ }^{\dagger} p<0.05$

ADHD: attention deficit hyperactivity disorder; SRBD: sleep-related breathing disorder; RLS: restless legs syndrome

was 42.5-87.8. Out of the 110 boys, 5 had a CPRS inattention score $>65$ (clinical cut-off) and 22 had a CPRS hyperactivity/ impulsivity score $>65$ (clinical cut-off).

Using the whole sample $(n=110)$, regression analysis revealed that none of the four sleep scales was associated with the CPRS inattention scale (Table I). However, a greater SRBD score was significantly associated with a higher CPRS hyperactivity/ impulsivity score $(p<0.005)$. Greater sleepiness during daytime was significantly associated with a lower CPRS hyperactivity/ impulsivity score ( $p=0.049$; Table I). To determine whether sleep scales are uniquely related to ADHD features, further regression analysis showed that none of the four sleep scores was associated with the CPRS opposition score (Table I).

Repeat regression analysis after excluding the five boys with a CPRS inattention score $>65$ similarly revealed no relationship between the four sleep scales and the CPRS inattention score ( $p>0.05$ ). Moreover, on excluding the 22 subjects with a CPRS hyperactivity/impulsivity score $>65$, repeat regression analysis again showed the same significant relationship between the SRBD score and the CPRS hyperactivity/impulsivity score $(p=0.032)$. However, the significant relationship between daytime sleepiness and the CPRS hyperactivity/impulsivity score was no longer seen in this subgroup $(p=0.532)$.

\section{DISCUSSION}

Although a wealth of studies has conveyed the consensus that parents of children with ADHD are more likely to report sleep problems in their children than parents of control children, ${ }^{(8,17-19)}$ such research is limited to the Western population. To the best of our knowledge, our study is the first to report the relationship between sleep-related behaviours and the cardinal features of ADHD in a Chinese population. Unlike existing studies that focused on extreme samples (i.e. normal vs. ADHD subjects), ${ }_{,}^{(8,17-20)}$ our study investigated such a relationship in a normal sample of male preschoolers, revealing that a worse SRBD score was associated with a higher level of hyperactivity/impulsivity. This underpins the importance of understanding the relationship between sleep-related behaviours and ADHD characteristics before the usual age of clinical diagnosis in children with ADHD.

In our nonclinical sample, we found a significant association between SRBD and hyperactivity/impulsivity, which is consistent with previous reports in clinical samples 
of children with ADHD. ${ }^{(20,21)}$ Konofal et al found that children with ADHD had more difficulties in breathing during sleep as compared to normal children. ${ }^{(22)}$ Other studies have also reported that sleep-disordered breathing was associated with behavioural problems, in particular hyperactivity and ADHD. ${ }^{(19,23)}$ Ali et al suggested that up to one-third of all children with sleep-disordered breathing show significant hyperactivity. ${ }^{(24)}$ The same authors also found that children with ADHD who had undergone adenotostillectomy for sleep-disordered breathing showed improvement in inattentive and hyperactive behaviours. ${ }^{(25)}$ However, the association between sleep-disordered breathing and hyperactive behaviour is found not only in children with ADHD, but also in those with mild hyperactive behaviours ${ }^{(21)}$ and in nonclinical samples, as shown in our study. The mechanisms by which SRBD may contribute to hyperactivity are yet unclear. It is possible that SRBD results in inadequate sleep duration, and fragmented or disrupted sleep that can lead or contribute to alterations in the neurochemical substrate of the prefrontal cortex, with resultant problems in mood, attention and behaviour. ${ }^{(17,26)}$

Current research on the association between RLS and ADHD symptoms is largely limited to clinical samples. A number of studies have shown that children with ADHD are more likely to have periodic limb movement disorder (PLMD) in sleep, an increased number of periodic limb movements in sleep (PLMS) or RLS. ${ }^{(5,20,27-31)}$ The possible mechanisms underlying the relationship between RLS and ADHD remain unknown, and our study did not find any association between RLS and the cardinal features of ADHD. This may partially be due to our study's use of a nonclinical sample. We postulate that PLMD, PLMS or RLS may occur in a select group of children with more severe ADHD symptoms, and may in fact represent a distinct categorical subset of ADHD. Moreover, the absence of a direct link between RLS and ADHD features in our sample also suggests that PLMD, PLMS or RLS may not directly lead to symptoms of ADHD through the mechanism of sleep disruption, but rather exacerbate ADHD in children. Epidemiological studies are required to further assess this postulation in large-scale nonclinical samples.

There were several strengths in this study. First, to the best of our knowledge, this is the first study reporting the relationship between sleep and the cardinal features of ADHD in normal preschool Chinese children. Second, even though our sample size was relatively small, our study results were derived only from boys within a narrow age range. As the clinical picture of ADHD changes with age, some of the manifestations of ADHD (e.g. hyperactivity) decrease or even disappear in some children as they grow older, while more than $50 \%$ of children with ADHD grow up to become adults with significant ADHDrelated symptoms. ${ }^{(12)}$ Since childhood is a critical developmental period, restricting the age range of participants in the study was important. Moreover, ADHD, which is more prevalent in boys than in girls, has clinical manifestations that differ between boys and girls. ${ }^{(5,12,13,32)}$ Hence, potential moderation effects due to age and gender were minimised in our study.

In terms of limitations, our study incorporated only the subjective measures obtained from maternal reports. Instrumental objective sleep measures can be obtained for further investigations of the relationship between sleep physiopathology and cardinal features of ADHD in Chinese preschoolers. Moreover, our findings may not be generalisable to children of other age ranges.

In conclusion, this study presents the association of sleeprelated behaviours and cardinal features of ADHD in a nonclinical Chinese sample. Together with existing evidence derived from clinical ADHD samples, our findings suggest that RLS may occur in a select group of children with more severe ADHD symptoms and that SRBD occurs across the entire spectrum of hyperactivity from the preclinical stage of ADHD.

\section{REFERENCES}

1. Polanczyk G, de Lima MS, Horta BL, Biederman J, Rohde LA. The worldwide prevalence of ADHD: a systematic review and metaregression analysis. Am J Psychiatry 2007; 164:942-8.

2. American Psychiatric Association. Diagnostic and Statistical Manual of Mental Disorders: DSM-IV. 4th ed. Washington (DC): American Psychiatric Association, 1994.

3. Oguzturk O, Ekici M, Cimen D, Ekici A, Senturk E. Attention deficit/ hyperactivity disorder in adults with sleep apnea. J Clin Psychol Med Settings 2013; 20:234-9.

4. Kirov R, Uebel H, Albrecht B, et al. Attention-deficit/hyperactivity disorder (ADHD) and adaptation night as determinants of sleep patterns in children. Eur Child Adolesc Psychiatry 2012; 21:681-90.

5. Sadeh A, Pergamin L, Bar-Haim Y. Sleep in children with attention-deficit hyperactivity disorder: a meta-analysis of polysomnographic studies. Sleep Med Rev 2006; 10:381-98.

6. Gaultney JF, Terrell DF, Gingras JL. Parent-reported periodic limb movement, sleep disordered breathing, bedtime resistance behaviors, and ADHD. Behav Sleep Med 2005; 3:32-43.

7. Marcotte AC, Thacher PV, Butters M, et al. Parental report of sleep problems in children with attentional and learning disorders. J Dev Behav Pediatr 1998; 19:178-86.

8. van der Heijden KB, Smits MG, Gunning WB. Sleep-related disorders in ADHD: a review. Clin Pediatr (Phila) 2005; 44:201-10.

9. Scott N, Blair PS, Emond AM, et al. Sleep patterns in children with ADHD: a population-based cohort study from birth to 11 years. J Sleep Res 2013; 22:121-8.

10. Shur-Fen Gau S. Prevalence of sleep problems and their association with inattention/hyperactivity among children aged 6-15 in Taiwan. J Sleep Res 2006; 15:403-14.

11. Wu YW, Xing G, Fuentes-Afflick E, et al. Racial, ethnic, and socioeconomic disparities in the prevalence of cerebral palsy. Pediatrics 2011; 127:e674-81.

12. Biederman J, Faraone SV. Attention-deficit hyperactivity disorder. Lancet 2005; 366:237-48.

13. Biederman J, Mick E, Faraone SV, et al. Influence of gender on attention deficit hyperactivity disorder in children referred to a psychiatric clinic. Am J Psychiatry 2002; 159:36-42.

14. Arnold LE, Elliot M, Sachs L, et al. Effects of ethnicity on treatment attendance, stimulant response/dose, and 14-month outcome in ADHD. J Consult Clin Psychol 2003; 71:713-27.

15. Gau SS, Soong WT, Chiu YN, Tsai WC. Psychometric properties of the Chinese version of the Conners' Parent and Teacher Rating Scales-Revised: Short Form. J Atten Disord 2006; 9:648-59.

16. Wang $\mathrm{CH}$, Yang CM, Huang YS. The Validation and Reliability of Chinese Version of the Pediatric Sleep Questionnaire for Patients with Sleep Breathing Problem. Taiwan J Psychiatry 2012; 26:177-86.

17. Owens JA. The ADHD and sleep conundrum: a review. J Dev Behav Pediatr 2005; 26:312-22. 
18. Cohen-Zion M, Ancoli-Israel S. Sleep in children with attention-deficit hyperactivity disorder (ADHD): a review of naturalistic and stimulant intervention studies. Sleep Med Rev 2004; 8:379-402.

19. Corkum P, Tannock R, Moldofsky H. Sleep disturbances in children with attention-deficit/hyperactivity disorder. J Am Acad Child Adolesc Psychiatry 1998; 37:637-46.

20. Silvestri R, Gagliano A, Arico I, et al. Sleep disorders in children with attention-deficit/hyperactivity disorder (ADHD) recorded overnight by video-polysomnography. Sleep Med 2009; 10:1132-8.

21. O'Brien LM, Holbrook CR, Mervis CB, et al. Sleep and neurobehavioral characteristics of 5- to 7-year-old children with parentally reported symptoms of attention-deficit/hyperactivity disorder. Pediatrics 2003; 111:554-63.

22. Konofal E, Lecendreux M, Cortese S. Sleep and ADHD. Sleep Med 2010; 11:652-8.

23. Owens JA, Maxim R, Nobile C, McGuinn M, Msall M. Parental and selfreport of sleep in children with attention-deficit/hyperactivity disorder. Arch Pediatr Adolesc Med 2000; 154:549-55.

24. Ali NJ, Pitson DJ, Stradling JR. Snoring, sleep disturbance, and behaviour in 4-5 year olds. Arch Dis Child 1993; 68:360-6.

25. Ali NJ, Pitson D, Stradling JR. Sleep disordered breathing: effects of adenotonsillectomy on behaviour and psychological functioning. Eur J Pediatr 1996; 155:56-62.

26. Beebe DW, Gozal D. Obstructive sleep apnea and the prefrontal cortex towards a comprehensive model linking nocturnal upper airway obstruction to daytime cognitive and behavioral deficits. J Sleep Res 2002; 11:1-16.

27. O'Brien LM, Ivanenko A, Crabtree VM, et al. Sleep disturbances in children with attention deficit hyperactivity disorder. Pediatr Res 2003. 54:237-43.

28. Chervin RD, Archbold KH. Hyperactivity and polysomnographic findings in children evaluated for sleep-disordered breathing. Sleep 2001; 24:313-20.

29. Picchietti DL, Underwood DJ, Farris WA, et al. Further studies on periodic limb movement disorder and restless legs syndrome in children with attention-deficit hyperactivity disorder. Mov Disord 1999; 14:1000-7.

30. Konofal E, Cortese S. Restless legs syndrome and attention-deficit/ hyperactivity disorder. Ann Neurol 2005; 58:341-2.

31. Cortese S, Konofal E, Lecendreux $M$, et al. Restless legs syndrome and attention-deficit/hyperactivity disorder: a review of the literature. Sleep 2005; 28:1007-13.

32. Qiu A, Crocetti D, Adler M, et al. Basal ganglia volume and shape in children with attention deficit hyperactivity disorder. Am J Psychiatry 2009; 166:74-82.

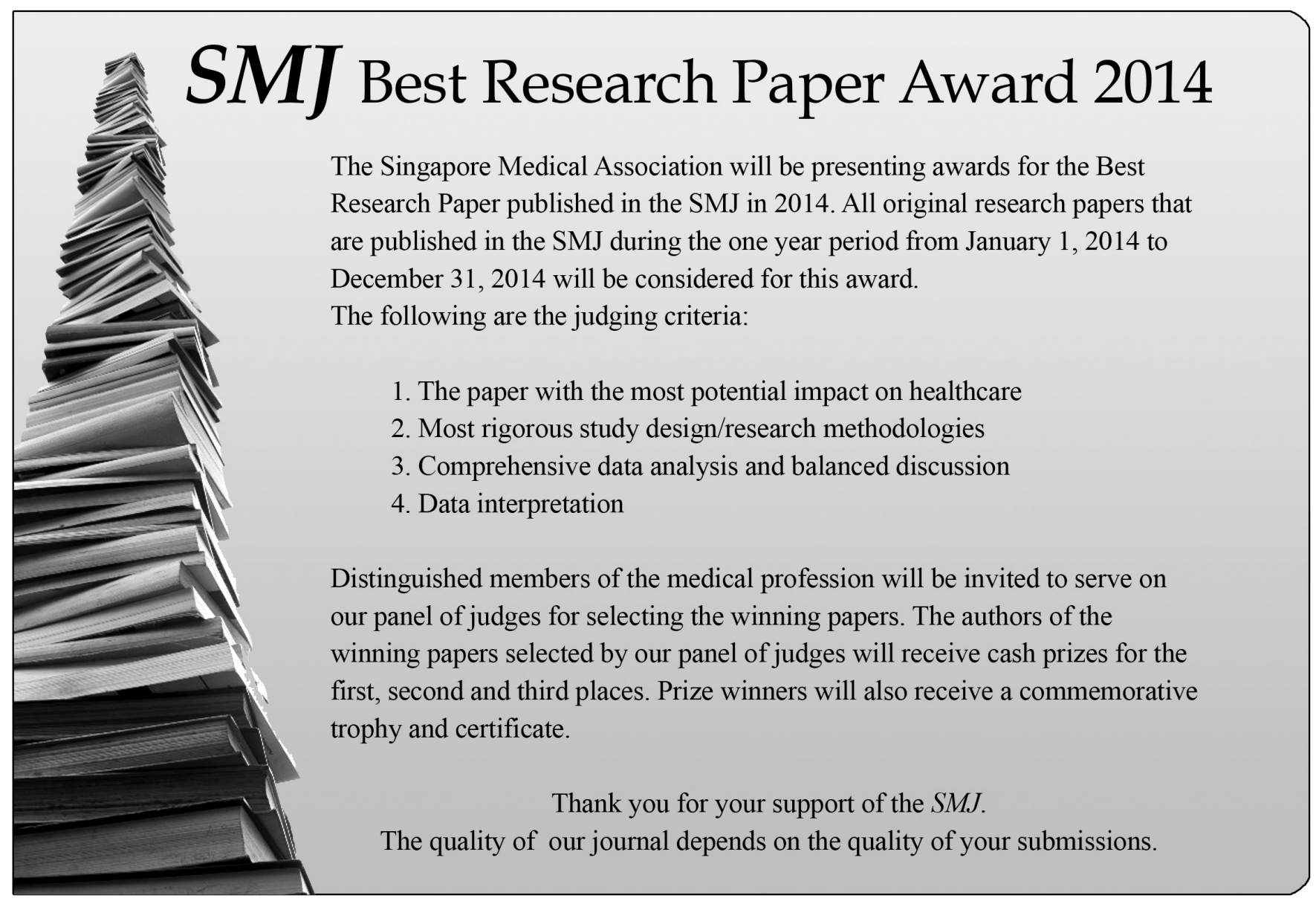

